\title{
Diagnostic value of linked color imaging based on endoscopy for gastric intestinal metaplasia: a systematic review and meta- analysis
}

\author{
Xiaochuang Shu ${ }^{1,2,3 \#}$, Guozhi Wu ${ }^{1,2,3 \#}$, Yanjun Zhang ${ }^{4 \#}$, Yuping Wang ${ }^{2,3}$, Ya Zheng ${ }^{2,3}$, Qinghong Guo ${ }^{2,3}$, \\ Rui Ji ${ }^{2,3}$, Yongning Zhou ${ }^{2,3}$ \\ ${ }^{1}$ The First Clinical Medical College of Lanzhou University, Lanzhou, China; ${ }^{2}$ Department of Gastroenterology, The First Hospital of Lanzhou \\ University, Lanzhou, China; ${ }^{3}$ Key Laboratory for Gastrointestinal Diseases of Gansu Province, Lanzhou University, Lanzhou, China; ${ }^{4}$ General \\ Internal Medicine Department of Donggang Branch, The First Hospital of Lanzhou University, Lanzhou, China \\ Contributions: (I) Conception and design: X Shu, G Wu, Y Zhang; (II) Administrative support: R Ji, Y Zhou; (III) Provision of study materials \\ or patients: Y Wang; (IV) Collection and assembly of data: X Shu, G Wu, Y Zhang; (V) Data analysis and interpretation: Y Zheng, Q Guo; (VI) \\ Manuscript writing: All authors; (VII) Final approval of manuscript: All authors. \\ "These authors contributed equally to this work. \\ Correspondence to: Prof. Yongning Zhou. Department of Gastroenterology, Key Laboratory for Gastrointestinal Diseases of Gansu Province, The \\ First Hospital of Lanzhou University, No.1 West Donggang Road, Lanzhou 730000, China. Email: zhouyn@lzu.edu.cn; Prof. Rui Ji. Department \\ of Gastroenterology, Key Laboratory for Gastrointestinal Diseases of Gansu Province, The First Hospital of Lanzhou University, No.1 West \\ Donggang Road, Lanzhou 730000, China. Email: jir@lzu.edu.cn.
}

Background: The diagnostic value of linked color imaging based on endoscopy for gastric intestinal metaplasia has shown variable results. Therefore, this meta-analysis sought to systematically evaluate the value of linked color imaging (LCI) based on the blue laser endoscopy system for the diagnosis of gastric intestinal metaplasia (GIM).

Methods: Literature searches were conducted of electronic databases including PubMed, Embase, the Cochrane Library, and Web of Science to screen diagnostic tests of LCI. The random-effects model was adopted to calculate the diagnostic efficacy of LCI for GIM. Meta-DiSc 1.40 software was applied for the calculation of sensitivity, specificity, and likelihood ratios; symmetric receiver operator characteristic (SROC) curves were drawn, and the areas under the SROC curves (AUCs) were computed. Quality of the included studies was chosen to assess using the quality assessment of diagnostic accuracy studies-2 (QUADAS-2) tool. Results: Six original studies involving 700 participants were included in the meta-analysis. The pooled sensitivity, specificity, positive likelihood ratio, and negative likelihood ratio of LCI for diagnosing GIM were 0.87 (0.83-0.91), 0.86 (0.82-0.89), 5.72 (3.63-8.99), and $0.17(0.08-0.36)$, respectively. SROC curve analysis showed that the AUC value was 0.9283 .

Discussion: Our study shows that LCI can be used for the accurate diagnosis of GIM. Considering weaknesses of available studies in terms of design, further studies with rigorous design are in need for further validating the findings of this meta-analysis.

Keywords: Endoscopy; linked color imaging (LCI); gastric intestinal metaplasia (GIM); diagnosis; meta-analysis

Submitted Feb 21, 2021. Accepted for publication Mar 23, 2021.

doi: 10.21037/atm-21-1051

View this article at: http://dx.doi.org/10.21037/atm-21-1051 


\section{Introduction}

Gastric intestinal metaplasia (GIM) is considered to be a precursor to the development of dysplasia and intestinal gastric adenocarcinoma. Multiple studies have described GIM as an independent risk factor for gastric cancer (1-4). Currently, the diagnosis of GIM mainly depends on the histological study of biopsy specimens. As GIM typically originates in flat mucosa and results in few morphological changes, the ability of white light endoscopy to detect it is limited. Narrow-band imaging (NBI), an image-enhanced endoscopic method, has increased the detection rate of GIM (5-10). However, clear diagnostic criteria have yet to be established, which brings difficulties for clinical doctors in identifying GIM lesions.

LASEREO, a blue laser endoscope system officially launched by FUJIFILM Corporation (Tokyo, Japan) in 2012, uses a laser light source to render brighter and clearer endoscopic images with more identifiable layers $(11,12)$. The linked color imaging (LCI) mode of this system can facilitate special processing of images to obtain greater color contrast (13), which is conducive to the identification of lesions, thereby improving the efficiency of targeted biopsy of suspicious lesions and, consequently, the accuracy of GIM diagnosis. In recent years, prospective studies have explored the effectiveness of LCI for the diagnosis of GIM. In the present study, we aimed to evaluate the value of LCI for the diagnosis of GIM and provide medical evidence supporting the clinical application of LCI by conducting a meta-analysis of LCIrelated literature. We present the following article in accordance with the PRISMA reporting checklist (available at http://dx.doi.org/10.21037/atm-21-1051).

\section{Methods}

\section{Search strategy}

Online searches were conducted of electronic databases including PubMed, Embase, the Cochrane Library, and Web of Science. The search time was set from database establishment to February 7, 2021. Manual searches were also performed to identify articles of potential interest from the references of retrieved studies. In terms of the search strategy, we combined theme words with free words and divided the topics of the studies into 3 aspects: the target disease, the tests to be evaluated, and diagnostic accuracy indexes. The search terms included "Metaplasia" and "Linked color imaging" as well as their corresponding abbreviations. The language was restricted to English. Specific details of the search strategies are described in the Supplementary Materials (Appendix 1).

\section{Inclusion and exclusion criteria}

The inclusion criteria for studies were as follows: (I) sufficient information was provided to enable the establishment of a $2 \times 2$ table to determine the number of false positive, true positive, false negative, and true negative cases; (II) the diagnostic test to be evaluated was LCI examination, with the golden criterion being that pathological biopsy could confirm the existence of GIM; and (III) English was the publication language. There were no restrictions in terms of the age or sex of study participants, or sample size.

The exclusion criteria were as follows: (I) duplicated data sources; (II) study participants had clear risk factors or had already been diagnosed with GIM; (III) the diagnostic tests applied did not meet the golden criterion as the means of diagnosis; and (IV) complete 4-table data could not be extracted. Literature reviews, letters, meeting abstracts, case reports, and animal studies were also excluded.

\section{Literature screening and data extraction}

X Shu and G Wu screened the retrieved literature and extracted relevant data according to preset inclusion and exclusion criteria. Any disagreements that arose were resolved through discussions with a $3^{\text {rd }}$ author. Data extracted from each study mainly included the following information: first author, publishing year, country, study design, object of research, sample size, screening methods, and test parameters (true positive, false positive, false negative, and true negative).

\section{Risk-of-bias assessment in the included studies}

The QUADAS-2 scale, a quality assessment tool for diagnostic tests, was chosen to assess the risk of bias (14). The scale comprises 2 parts: assessment of risk of bias and applicability. The risk-of-bias assessment is composed of 4 aspects: case selection, tests to be evaluated, the golden criterion as well as case flow and progress. The applicability assessment covers 3 aspects: case selection, tests to be evaluated, and the golden criterion. In each aspect, the risk of bias was classified as "high", "low", or "unclear". 


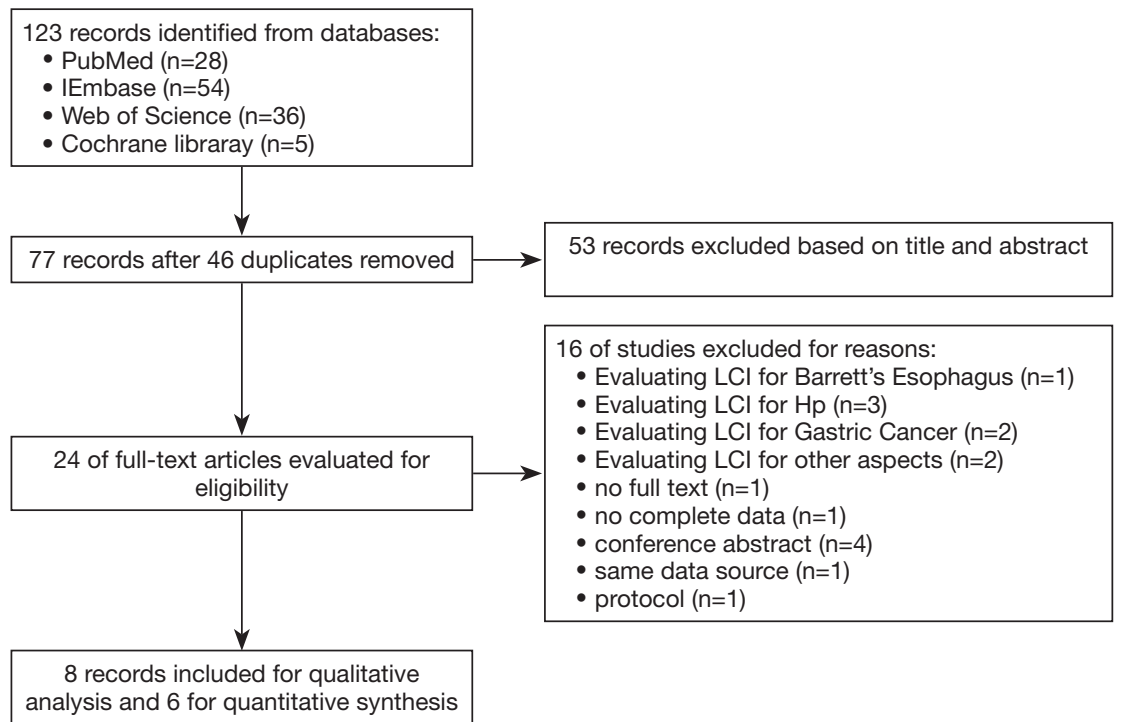

Figure 1 Flow diagram of the literature screening process.

\section{Data integration and statistical analysis}

Heterogeneity in diagnostic tests often results from threshold effects and non-threshold effects. First, to judge whether there was heterogeneity caused by threshold effects, the Spearman's correlation coefficient was calculated. If $\mathrm{P}<0.05$, threshold effects were considered to exist and the data were not integrated. Statistical heterogeneity was evaluated using the $\chi^{2}$ test and $\mathrm{I}^{2}$ statistics. The value range of $\mathrm{I}^{2}$ was $0 \%$ (no heterogeneity) to $100 \%$ (maximum level of heterogeneity). If $\mathrm{I}^{2}>50 \%$, a significant level of heterogeneity was considered to exist. The DerSimonian and Laird random-effects method was selected for data integration and the pooled sensitivity, specificity, likelihood ratio, and diagnostic odds ratio (DOR) as well as the corresponding $95 \%$ confidence intervals (CIs) based on perpatient were computed. A statistically significant difference was indicated by $\mathrm{P}<0.05$. As there were fewer than 10 studies included in the meta-analysis, publication bias testing and meta-regression analysis were not performed to analyze heterogeneity triggered by non-threshold effects. Sensitivity analysis was carried out to evaluate the stability of the results. Revman (version 5.3) and Meta-DiSc (version 1.40) were used to perform the statistical analyses.

\section{Results}

\section{Results of literature screening and bias risk assessment}

The initial search yielded 123 articles including 28 in
PubMed, 54 in Embase, 5 in the Cochrane Library, and 36 in Web of Science. After the completion of title and abstract screening and full-text reading, 7 research articles (15-21) and 1 conference abstract (22) were selected. However, Fukuda et al.'s (21) study did not report the number of subjects (16); instead, the analysis was conducted according to the number of lesions. Furthermore, in Min et al.'s (19) and Ono et al.'s (20) studies, analyses were also based on the number of lesions. The Spearman's correlation coefficient was calculated, and suggested significant threshold effects in the results of the above studies; consequently, Min et al.'s (19), Ono et al.'s (20) and Fukuda et al.'s (21) trials were excluded from our meta-analysis. Because the quality of conference literature could not be evaluated, the literature of this category was only included for sensitivity analysis. Ultimately, 6 original studies involving 700 participants were included. The specific literature screening process and the baseline status of the included studies are detailed in Figure 1 and Table 1, respectively. The results of bias evaluation are shown in Figure 2.

\section{Heterogeneity testing}

By calculating the Spearman's correlation coefficient between the logarithm of sensitivity and the logarithm of (1-specificity), we arrived at $\mathrm{P}=0.544$, indicating the absence of a threshold effect. The pooled DOR, Cochran-Q, degree of freedom (df), and $\mathrm{I}^{2}$ value were $38.21,12.75,5$ $(\mathrm{P}=0.00258)$, and $60.8 \%$, respectively. The above results 
Table 1 Characteristics of the included studies

\begin{tabular}{|c|c|c|c|c|c|c|c|c|}
\hline Study & Country & $\begin{array}{l}\text { Participants } \\
\text { (M/F) }\end{array}$ & $\begin{array}{l}\text { Mean age } \\
\text { (years) }\end{array}$ & Trial design & Endoscopy type & Observed site & $\begin{array}{l}\text { Endoscopic } \\
\text { term }\end{array}$ & Basis \\
\hline $\begin{array}{l}\text { Lee et al. } \\
2020 \text { (15) }\end{array}$ & China & $100(52 / 48)$ & 51.2 & $\begin{array}{l}\text { Single-center, } \\
\text { prospective }\end{array}$ & EG-760R, $760 Z$ & Antrum & - & Pre-patient \\
\hline $\begin{array}{l}\text { Chen et al. } \\
2019 \text { (16) }\end{array}$ & China & $107(64 / 43)$ & 53.5 & $\begin{array}{l}\text { Single-center, } \\
\text { prospective }\end{array}$ & LCl endoscopy & Antrum & PLC $^{\ddagger}$ & $\begin{array}{l}\text { Pre-patient/ } \\
\text { pre-lesion }\end{array}$ \\
\hline $\begin{array}{l}\text { Zhang et al. } \\
2021 \text { (18) }\end{array}$ & China & $277(156 / 121)$ & 53.3 & $\begin{array}{l}\text { Single-center, } \\
\text { prospective }\end{array}$ & $\begin{array}{c}\text { EG-L590ZW, } \\
\text { L700ZW, L580NW }\end{array}$ & $\begin{array}{l}\text { Less, Gre", } \\
\text { angulus }\end{array}$ & PLC $^{\ddagger}$ & Pre-patient \\
\hline $\begin{array}{l}\text { Min et al. } \\
2019 \text { (19) }\end{array}$ & China & $63(36 / 27)$ & 54.2 & $\begin{array}{l}\text { Single-center, } \\
\text { prospective }\end{array}$ & GF-L590WR & $\begin{array}{c}\text { Antrum, corpus, } \\
\text { angulus }\end{array}$ & $\mathrm{PIM}^{\S}$ & $\begin{array}{l}\text { Pre-patient/ } \\
\text { pre-lesion }\end{array}$ \\
\hline
\end{tabular}

${ }^{\dagger}$, LCS, Lavender color sign; ${ }^{\ddagger}$, PLC, Patchy Lavender Color; ${ }^{\S}$, PIM, Purple in Mist; ${ }^{\Uparrow}$, less, the lesser curvature of the gastric antrum and corpus; Gre, the greater curvature of the gastric antrum and corpus.

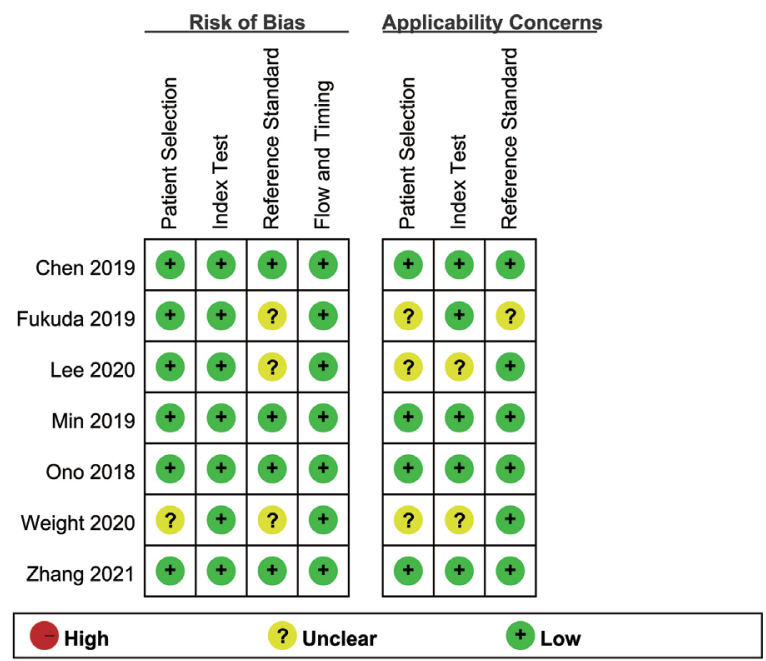

Figure 2 Risk of bias assessment.

suggested the existence of heterogeneity caused by nonthreshold effects; consequently, a random-effects model was applied for data integration in this meta-analysis.

\section{Diagnostic value of LCI for GIM}

The pooled sensitivity, specificity, positive likelihood ratio, and negative likelihood ratio of LCI for the diagnosis of
GIM were $0.87(0.83-0.91), 0.86(0.82-0.89), 5.72$ (3.63$8.99)$, and $0.17(0.08-0.36)$, respectively (Figure $3 A, B, C, D)$. Symmetric receiver operator characteristic (SROC) curve analysis revealed an AUC value of 0.9283 (Figure 4).

\section{Sensitivity analysis}

With the exclusion of Lee et al.'s (15) study, the heterogeneity of the combined sensitivity and negative likelihood ratio was significantly reduced. With the exclusion of Chen et al.'s (16) study, the heterogeneity of the combined specificity and positive likelihood ratio was significantly reduced. When the above 2 studies were excluded simultaneously, the pooled DOR was 74.83 (41.44, 135.09), Cochran-Q was 1.63 , df was $3(\mathrm{P}=0.6528)$, and $\mathrm{I}^{2}$ was $0.0 \%$, At this point, the sensitivity, specificity, positive likelihood ratio, negative likelihood ratio, and AUC value were 0.90 (0.85-0.93), 0.89 (0.84-0.92), 7.47 (5.18-10.78), $0.12(0.08-0.20)$, and 0.9536 , respectively. No obvious change was observed in the combined results before and after the inclusion of Wu et al.'s (22) conference abstract. There was no significant change in the results after data integration using the fixed-effects model.

\section{Discussion}

According to the criteria of the updated Sydney System, 
A
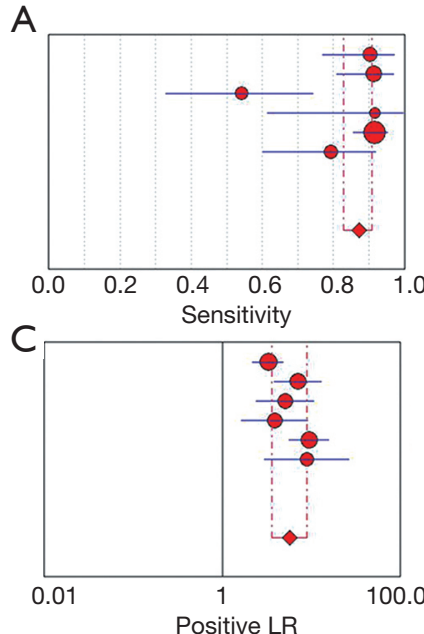
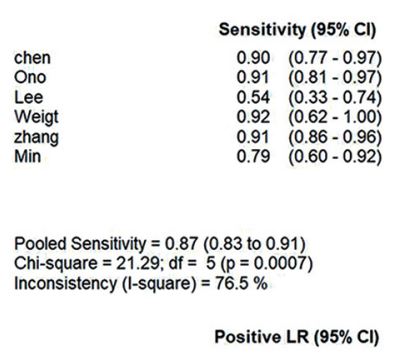
$\begin{array}{lll}\text { chen } & 3.31 & (2.20-4.97) \\ \text { Ono } & 7.11 & (3.84-13.15)\end{array}$ Lee $\quad 5.15(2.43-10.91)$ $\begin{array}{lll}\text { Weigt } & 3.90 & (1.63-9.33) \\ \text { zhang } & 9.57 & (5.69-16.09)\end{array}$ $\begin{array}{lll}\text { Mhang } & 9.57 & (5.69-16.09) \\ \text { Min } & 8.99 & (3.00-26.91)\end{array}$

Random Effects Model Pooled Positive $L R=5.72$ (3.63 to 8.99$)$ Cochron $=14.39, \mathrm{~d}=5(\mathrm{p}=0.0133)$ Tau-squared $=0.1963$

$B$
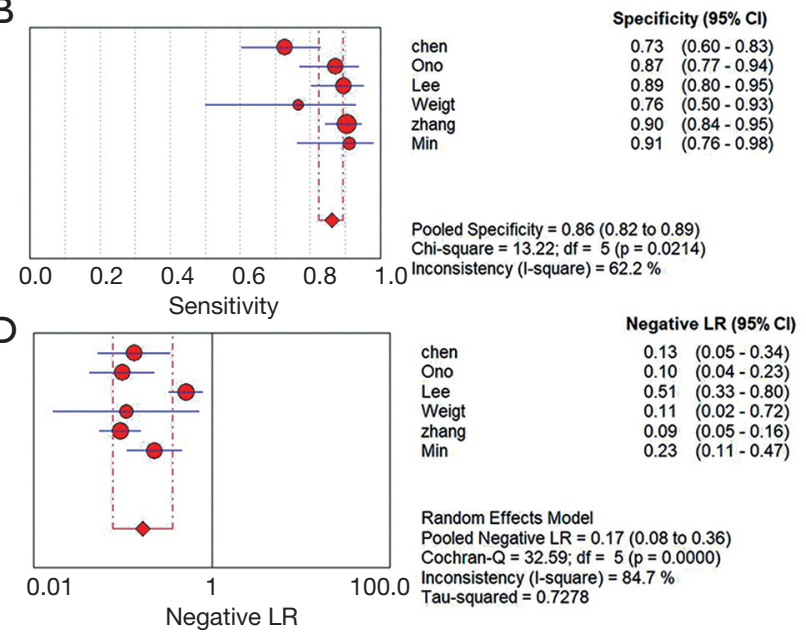

Figure 3 Pooled results of included studies. Pooled sensitivity (A), specificity (B), positive likelihood ratio (C), and negative likelihood ratio (D). df, degree of freedom; LR, likelihood ratio; CI, confidence intervals.

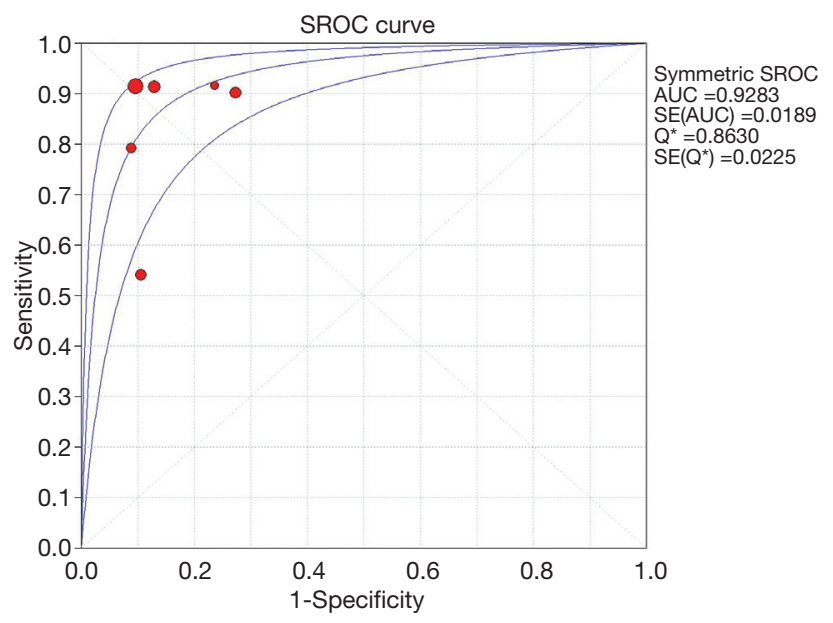

Figure 4 SROC curve and AUC. SE, standard error; $\mathrm{Q}^{*}$, the point of the curve in which sensitivity equals specificity; SROC, symmetric receiver operator characteristic; AUC, area under curve.

pathological biopsy is still the golden criterion for the diagnosis of GIM. However, since GIM can form in different parts of the stomach, lesions are likely to be missed by random biopsy sampling. Consequently, initial identification of GIM lesions under endoscopy has great diagnostic significance. The blue laser endoscopy system that has appeared in recent years has performed well in diagnostic efficiency. Many studies have confirmed that by observing "PLC" (patchy lavender color with a regular mucosal pattern and a clear border) (16,18), "PIM" (Purple in Mist; purple mixed with white on the epithelium with signs of mist detected by the non-magnifying LCI observation) (19), or "LCS" (20) (a lavender color sign), along with other signs through LCI, the detection rate of GIM will significantly increase. In this paper, the sensitivity, specificity, and positive and negative likelihood ratios of LCI for the diagnosis of GIM were integrated through a systematic search of relevant literature, and an SROC curve was drawn to provide visual evidence of the diagnostic efficacy of LCI.

The results of our meta-analysis showed that the combined sensitivity of LCI for the diagnosis of GIM was $0.87(0.83-0.91)$, the specificity was $0.86(0.82-0.89)$, the positive likelihood ratio was $5.72(3.63-8.99)$, and the negative likelihood ratio was $0.17(0.08-0.36)$, with the AUC value being 0.9283 . Our results have confirmed for the first time that LCI has good diagnostic efficacy for GIM. Previous studies on the detection of GIM through endoscopy systems have mainly focused on the assessment of the diagnostic capability of NBI for GIM (23). Although our results did not reveal much of a difference between the diagnostic performance of NBI and that of LCI, there were still substantial differences in the diagnostic criteria for GIM in the tests included in the above-mentioned studies, which inevitably led to the existence of heterogeneity caused by hidden threshold effects. Also, the light blue crest and white opaque substance observed with NBI were of high specificity and positive predictive value for the diagnosis of GIM (24,25); although, when observed by NBI, the gastric 
cavity was slightly dark in color overall. To obtain accurate markings, available equipment for the optical magnification of endoscopy must be used $(26,27)$. Although the diagnosis rate of magnified endoscopy combined with NBI for GIM can reach over $90 \%$ (28), due to the fact that magnified endoscopy requires more examination time, richer experience, and higher cost, this technique is not widely applied in clinical practice. Therefore, LCI has emerged as a new means for diagnosing GIM. Compared with NBI, LCI offers greater emphasis of mucous membrane color changes. Furthermore, due to its sufficient brightness, LCI can visualize the purple changes in the gastric cavity which are characteristic of GIM $(14,19)$, which is of considerable value for diagnosing GIM.

In our sensitivity analysis, we also found that after the exclusion of Lee et al. (15) and Chen et al.'s (16) studies, the heterogeneity among the studies was lowered tremendously. Lee $e t a l$. focused on the diagnostic value of LCI for Helicobacter pylori, with the diagnosis rate of GIM used only as a secondary outcome indicator. Therefore, a remarkable bias in subject group selection was inevitable, which affects the accuracy of the results. Also, Lee et al. believed that GIM should be taken into consideration when uneven and irregular white plaques were found under endoscopy; this differed from other studies, which identified lavender lesions as a sign of GIM. The inconsistency in diagnostic criteria also led to heterogeneity induced by potential threshold effects. In regard to Chen et al.'s report, considering that white light endoscopy and LCI were carried out during the same procedure in the research, the previous white light endoscopy observations might have led to deviations in the LCI examination results. After excluding Lee et al. and Chen $e t$ al.'s studies, the combined sensitivity, specificity, likelihood ratio, and AUC value of LCI for diagnosing GIM were all higher than before.

Our analysis has several limitations that cannot be ignored. First, due to the lack of relevant original studies and the fact that the included studies were almost all focused on single-center cohorts, there was a relatively small sample size for analysis, which rendered it impossible for us to perform subgroup analysis based on variables such as country and racial group. Second, each included study used different diagnostic criteria for the diagnosis of GIM with LCI, but essentially, the existence of GIM was considered when lavender changes were observed. Thirdly, the sensitivity results indicated instability in the overall results; nevertheless, when we excluded some studies and analyzed the sources of heterogeneity of the included studies, the summarized results revealed an improvement in the diagnostic efficacy of LCI. Finally, what was noteworthy was that in the studies of Fukuda et al., Ono et al., and Min et al., the calculations of true positive, true negative, false positive, and false negative test parameters were conducted according to the results of lesions. Due to the insufficient number of included studies, we did not synthesize data based on the number of lesions.

In summary, under conventional endoscopy, LCI, as a non-invasive inspection method, can detect unique purple mucosal changes, thus significantly improving the diagnostic efficiency and accuracy for GIM with no need for endoscopic magnification. These qualities make LCI convenient to guide targeted biopsy. In the future, LCI may become a novel imaging tool for monitoring patients with precancerous gastric lesions. For now, more high-quality, large-sample studies are required to further explore the role of LCI in the detection of precancerous gastric lesions.

\section{Acknowledgments}

Thanks to the Department of Gastroenterology at The First Hospital of Lanzhou University and the Key Laboratory for Gastrointestinal Diseases of Gansu Province for contributing to this meta-analysis.

Funding: This research was supported by the National Natural Science Foundation of China (71964021), the University Scientific Research Project of Gansu Province (2018B-011), the Fundamental Research Funds for the Central Universities (lzujbky-2020-kb16), and The Science and Technology Fund for Young Talents of Gansu Province (20JR5RA349).

\section{Footnote}

Reporting Checklist: The authors have completed the PRISMA reporting checklist. Available at http://dx.doi. org/10.21037/atm-21-1051

Conflicts of Interest: All authors have completed the ICMJE uniform disclosure form (available at http://dx.doi. org/10.21037/atm-21-1051). The authors have no conflicts of interest to declare.

Ethical Statement: The authors are accountable for all aspects of the work in ensuring that questions related to the accuracy or integrity of any part of the work are appropriately investigated and resolved. 
Open Access Statement: This is an Open Access article distributed in accordance with the Creative Commons Attribution-NonCommercial-NoDerivs 4.0 International License (CC BY-NC-ND 4.0), which permits the noncommercial replication and distribution of the article with the strict proviso that no changes or edits are made and the original work is properly cited (including links to both the formal publication through the relevant DOI and the license). See: https://creativecommons.org/licenses/by-nc-nd/4.0/.

\section{References}

1. Shichijo S, Hirata Y, Niikura R, et al. Histologic intestinal metaplasia and endoscopic atrophy are predictors of gastric cancer development after Helicobacter pylori eradication. Gastrointest Endosc 2016;84:618-24.

2. Lee TY, Wang RC, Lee YC, et al. The Incidence of Gastric Adenocarcinoma Among Patients With Gastric Intestinal Metaplasia: A Long-term Cohort Study. J Clin Gastroenterol 2016;50:532-7.

3. González CA, Sanz-Anquela JM, Companioni O, et al. Incomplete type of intestinal metaplasia has the highest risk to progress to gastric cancer: results of the Spanish follow-up multicenter study. J Gastroenterol Hepatol 2016;31:953-8.

4. Sakitani K, Hirata Y, Watabe H, et al. Gastric cancer risk according to the distribution of intestinal metaplasia and neutrophil infiltration. J Gastroenterol Hepatol 2011;26:1570-5.

5. Ang TL, Pittayanon R, Lau JYW, et al. A multicenter randomized comparison between high-definition white light endoscopy and narrow band imaging for detection of gastric lesions. Eur J Gastroenterol Hepatol 2015;27:1473-8.

6. Chiu PWY, Ng EKW, To KF, et al. Recognition of goblet cells upon endocytoscopy indicates the presence of gastric intestinal metaplasia. Dig Endosc 2014;26:52-6.

7. So J, Rajnakova A, Chan Y, et al. Endoscopic tri-modal imaging improves detection of gastric intestinal metaplasia among a high-risk patient population in Singapore. Dig Dis Sci 2013;58:3566-75.

8. Zhou Y, Zhou Z, Zhao J. Application of narrow band imaging-magnifying endoscopy in surveillance of gastric mucosal intestinal metaplasia. Chinese J Gastroenterol 2012;17:36-8.

9. Capelle LG, Haringsma J, de Vries AC, et al. Narrow Band Imaging for the Detection of Gastric Intestinal Metaplasia and Dysplasia During Surveillance Endoscopy.
Dig Dis Sci 2010;55:3442-8.

10. Dutta AK, Sajith KG, Pulimood AB, et al. Narrow band imaging versus white light gastroscopy in detecting potentially premalignant gastric lesions: a randomized prospective crossover study. Indian J Gastroenterol 2013;32:37-42.

11. Osawa H, Yamamoto H. Present and future status of flexible spectral imaging color enhancement and blue laser imaging technology. Dig Endosc 2014;26 Suppl 1:105-15.

12. Osawa H, Miura Y, Takezawa T, et al. Linked Color Imaging and Blue Laser Imaging for Upper Gastrointestinal Screening. Clin Endosc 2018;51:513-26.

13. Fukuda H, Miura Y, Hayashi Y, et al. Linked color imaging technology facilitates early detection of flat gastric cancers. Clin J Gastroenterol 2015;8:385-9.

14. Whiting PF, Rutjes AWS, Westwood ME, et al. QUADAS-2: a revised tool for the quality assessment of diagnostic accuracy studies. Ann Intern Med 2011;155:52936.

15. Lee SP, Lee J, Kae SH, et al. The role of linked color imaging in endoscopic diagnosis of Helicobacter pylori associated gastritis. Scand J Gastroenterol 2020;55:1114-20.

16. Chen $\mathrm{H}$, Wang $\mathrm{H}, \mathrm{Wu} \mathrm{X}$, et al. Predictability of gastric intestinal metaplasia by patchy lavender color seen on linked color imaging endoscopy. Lasers Med Sci 2019;34:1791-7.

17. Weigt J, Malfertheiner P, Canbay A, et al. Blue Light Imaging and Linked Color Imaging for the Characterization of Mucosal Changes in Chronic Gastritis: A Clinicians View and Brief Technical Report. Dig Dis 2020;38:9-14.

18. Zhang G, Zheng J, Zheng L, et al. Gastric intestinal metaplasia assessment between linked color imaging based on endoscopy and pathology. Scand J Gastroenterol 2021;56:103-10.

19. Min M, Dong TH, Liu Y, et al. Novel endoscopic findings as visualized by non-magnification endoscopy with linked color imaging are indicative of gastric intestinal metaplasia. Chinese Medical Journal 2019;132:782-8.

20. Ono S, Kato M, Tsuda M, et al. Lavender Color in Linked Color Imaging Enables Noninvasive Detection of Gastric Intestinal Metaplasia. Digestion 2018;98:222-30.

21. Fukuda H, Miura Y, Osawa H, et al. Linked color imaging can enhance recognition of early gastric cancer by high color contrast to surrounding gastric intestinal metaplasia. J Gastroenterol 2019;54:396-406.

22. Wu C, Vikneswaran N, Khor CJ, et al. A prospective randomized tandem gastroscopy study of linked color 


\section{Page 8 of 8}

imaging vs white light imaging for detection of upper gastrointestinal lesions. Gastroenterology 2020;158:S-557.

23. Wang L, Huang W, Du J, et al. Diagnostic yield of the light blue crest sign in gastric intestinal metaplasia: a metaanalysis. PloS One 2014;9:e92874.

24. An JK, Song GA, Kim GH, et al. Marginal turbid band and light blue crest, signs observed in magnifying narrowband imaging endoscopy, are indicative of gastric intestinal metaplasia. BMC Gastroenterol 2012;12:169.

25. Yao K, Iwashita A, Nambu M, et al. Nature of white opaque substance in gastric epithelial neoplasia as visualized by magnifying endoscopy with narrow-band imaging. Dig Endosc 2012;24:419-25.

26. Savarino E, Corbo M, Dulbecco P, et al. Narrowband imaging with magnifying endoscopy is accurate

Cite this article as: Shu X, Wu G, Zhang Y, Wang Y, Zheng Y, Guo Q, Ji R, Zhou Y. Diagnostic value of linked color imaging based on endoscopy for gastric intestinal metaplasia: a systematic review and meta-analysis. Ann Transl Med 2021;9(6):506. doi: 10.21037/atm-21-1051

\section{Shu et al. Meta-analysis of diagnostic value of LCI for GIM}

for detecting gastric intestinal metaplasia. World J Gastroenterol 2013;19:2668-75.

27. Yao K, Iwashita A, Tanabe H, et al. White opaque substance within superficial elevated gastric neoplasia as visualized by magnification endoscopy with narrowband imaging: a new optical sign for differentiating between adenoma and carcinoma. Gastrointest Endosc 2008;68:574-80.

28. Uedo N, Ishihara R, Iishi H, et al. A new method of diagnosing gastric intestinal metaplasia: narrowband imaging with magnifying endoscopy. Endoscopy 2006;38:819-24.

(English Language Editor: J. Reynolds) 


\section{Supplementary}

The Supplementary Materials of our meta-analysis can be obtained from the corresponding authors.

- Search strategy of PubMed

(((((Metaplasia[Title/Abstract]) OR (intestinal metaplasia[Title/Abstract])) OR (Gastric Intestinal Metaplasia[Title/ Abstract])) OR (IM[Title/Abstract])) OR (GIM[Title/Abstract])) OR ("Metaplasia"[Mesh])) AND ((linked color imaging[Title/Abstract]) OR (LCI[Title/Abstract]))

- Search strategy of Embase

\#1 'Metaplasia'/exp OR 'Metaplasia' OR 'intestinal metaplasia' OR 'Gastric Intestinal Metaplasia' OR 'IM' OR 'GIM':ti,ab \#2 'linked color imaging' OR 'LCI':ti,ab

\#3 \#1 AND \#2 